\title{
Testing Mindfulness-Based Relapse Prevention with Medications for Opioid Use Disorder Among Adults in Outpatient Therapy: a Quasi-experimental Study
}

\author{
Keith J. Zullig ${ }^{1}$ (D) $\cdot$ Laura R. Lander $^{2} \cdot$ Meghan Tuscano $^{3} \cdot$ Megan Garland $^{4} \cdot$ Gerry R. Hobbs $^{5} \cdot$ Laurel Faulkenberry $^{6}$
}

Accepted: 16 September 2021 / Published online: 11 October 2021

(C) The Author(s), under exclusive licence to Springer Science+Business Media, LLC, part of Springer Nature 2021

\begin{abstract}
Objectives This study aimed to explore the effectiveness of mindfulness-based relapse prevention (MBRP) with individuals receiving medication for opioid use disorder (MOUD) in a naturalistic, open-ended outpatient group treatment setting. Methods Eighty participants (mean age 36.3) who had at least 90 consecutive days substance free self-selected into treatment (MBRP, $n=35$ ) or comparison groups (treatment as usual, TAU, $n=45$ ). Outcomes tracked included treatment retention and relapse, and self-reported craving, anxiety, depression, and mindfulness at baseline, 12 weeks, 24 weeks, and 36 weeks postrecruitment. MBRP group participants attended biweekly 60-min sessions for 24 weeks. A linear mixed model analysis of variance determined the significance of the MBRP intervention on changes in craving, anxiety, depression, and mindfulness. Results No significant differences in sex, education level, insurance status, relationship status, or employment status were detected at baseline between groups. The 36-week retention (74\%, MBRP/MOUD; 71\%, TAU/MOUD) and relapse rates (43\%, MBRP/MOUD; 47\%, TAU/MOUD) were similar for the groups. There were only four relapses on opioids. Significant reductions $(p<.05)$ were observed in the MBRP/MOUD group for craving, anxiety, and depression in addition to significant increases in mindfulness compared to those in TAU/MOUD.

Conclusions Although state and federal resources are available to expand MOUD, no standard of behavioral therapy has been established as most complimentary to MOUD. The current study results suggest MBRP can be implemented as an outpatient therapy for individuals in MOUD.
\end{abstract}

Keywords MOUD $\cdot$ MBRP $\cdot$ Relapse $\cdot$ Retention $\cdot$ Co-occurring disorders

Keith J. Zullig

kzullig@hsc.wvu.edu

1 Department of Social and Behavioral Sciences, West Virginia University School of Public Health, Morgantown, WV, USA

2 Department of Behavioral Medicine and Psychiatry and Rockefeller Neuroscience Institute, West Virginia University School of Medicine, Morgantown, USA

3 West Virginia University Research Corporation, Morgantown, USA

4 Department of Occupational and Environmental Health Sciences, West Virginia University School of Public Health, Morgantown, USA

5 Department of Statistics, West Virginia University, Morgantown, USA

6 Department of Behavioral Medicine and Psychiatry, West Virginia University School of Medicine, Morgantown, USA
West Virginia (WV) has led the United States (US) in drug overdose per capita deaths with pre-COVID-19 overdoses peaking in 2017 at a rate of 52 per 100,000 people (CDC, 2017). The greatest number of overdoses was due to opioid misuse (CDC, 2015). While 2018 marked the first year since 1999 that overdose death rates declined nationally and in WV (Hedegaard et al., 2020), the COVID-19 epidemic has reversed progress made, and current provisional estimates put the increases in overdose deaths from 2019 to 2020 at 29.4\% nationally and 45\% in WV (Ahmad et al., 2021). The rural nature of WV may contribute to the inadequate treatment resources at a time when they are needed most. For example, $55 \%$ of $\mathrm{WV}$ counties do not have a provider waivered to prescribed buprenorphine (Andrilla et al., 2019). Currently, buprenorphine is one of three FDA-approved medications for opioid use disorders. Medication for opioid use disorder (MOUD) is considered the treatment of choice for opioid use disorders in large part due to MOUD 
significantly reducing mortality rates and saving lives (Larochelle et al., 2018; Sordo, et al., 2017; Winstanley et al., 2019).

The 2015 Recovery Enhancement for Addiction Treatment Act (US Congress, 2015) increased access to MOUD with buprenorphine by allowing eligible practitioners to request approval to treat up to 275 patients. This had major implications for the expansion of MOUD at both the national and state level. While the Substance Abuse and Mental Health Services Administration (SAMHSA) endorses that MOUD be a combination of medication, counseling, and behavioral therapy, no specific recommendations as to what form of counseling or behavioral therapy is most effective for individuals with opioid use disorders presently exist. Furthermore, the research on the efficacy of behavioral interventions with individuals receiving MOUD is mixed. For example, Carroll and Weiss (2017) conducted a review of randomized controlled trials adding a behavioral intervention to MOUD and found four studies provided no additional benefit while four others demonstrated efficacy, with contingency management interventions having the most robust effect. A confounding factor in identifying effective interventions for individuals with substance use disorders is that approximately $49 \%$ of these individuals also have a co-occurring mental health diagnosis according to the 2019 National Survey on Drug Use and Health (SAMHSA, 2020). Thus, integrating and adapting effective evidencedbased treatments into MOUD which can target mental health symptoms in addition to SUD symptoms has the potential to improve intervention outcomes. Mindfulness-based interventions have been well established as an evidence-based intervention to improve mental health outcomes ( $\mathrm{Gu}$ et al., 2015).

A review of the efficacy of mindfulness interventions as an adjunctive treatment for substance use disorders suggests two primary models: Mindfulness-based relapse prevention (MBRP) (Bowen et al., 2006, 2014, 2017; Roos et al., 2019; Witkiewitz et al., 2014) and mindfulness-oriented recovery enhancement (MORE) (Garland, 2013; Garland et al., 2010, 2013). Conceptually, the core goals of MBRP are to cultivate internal and external awareness of substance use cues and triggers, attending mindfully to the discomforts the triggers elicit, and teaching targeted skills to cope with craving. By shifting the non-judging attention to the direct experience of sensations, thoughts, and emotions and away from the reactions, stories, and judgments of what are often painful experiences, the automatic and habitual reactions are broken, facilitating the recovery process. The MBRP model is more adaptable to an open-ended group, has a greater number of clinical trials to support its efficacy, and has been shown to be effective in a rolling group format (Korecki et al., 2020; Witkiewitz et al., 2014). The MORE model is less adaptable to an open-ended group in that sessions are designed to occur in succession and build on one another (Ivtzan, 2020). For our protocol design, we wanted to target both substance use disorder (SUD) and co-occurring mood disorders and there is evidence to suggest that MBRP can be more effective with individuals with co-occurring SUD and anxiety and depression (Roos et al., 2017).

The efficacy of mindfulness interventions as evidencebased adjunctive treatments for substance use disorders has been demonstrated (Bowen et al., 2014; Penberthy et al., 2015; Witkiewitz et al., 2013). Mindfulness-based relapse prevention (MBRP) has also been tested in a short-term residential treatment setting among individuals with differing SUD diagnoses (Roos et al., 2019). These researchers held rolling admission therapy groups twice each week for $1 \mathrm{~h}$ over 8 weeks for 8 total hours of programing. However, little research has been conducted to test the effectiveness of MBRP in a naturalistic, rolling admission outpatient setting for those receiving MOUD and for more than 12 weeks.

MBRP has not been shown to have greater effectiveness for the treatment of one particular substance versus another and most RCTs have targeted treatment of polysubstance use (Grant, 2015). MBRP is designed to be most effective after initial stabilization in treatment has been established, integrating mindfulness practices with cognitive-behavioral relapse prevention to maintain treatment gains and develop a lifestyle further supporting well-being and recovery. Given that opioid use disorder is a chronic relapsing disease with high risk of overdose and death, assessing models of treatment that can be adapted and administered for a longer duration is clinically indicated.

In a preliminary study, Zullig et al. (2018) reported that among completers of the 8-week MBRP/MOUD outpatient intervention, significant reductions were observed in reported depression and significant increases were observed in reported mindfulness when compared to those in treatment as usual (TAU) MOUD. However, this preliminary research was limited by its single group study design where participants served as their own controls (i.e., each participant participated in 8 weeks of TAU/MOUD before the 8-week MBRP/MOUD intervention phase began), followup measurement length, and small sample size. For example, although 32 participants were recruited to participate, only five completed the MBRP/MOUD intervention. Finally, study participants participated in the 8-week MBRP/MOUD intervention as a cohort limiting the researcher's ability to study how MBRP would function in an open-ended group treatment setting, which is the most common group type used in outpatient substance use disorder treatment.

The objective of the current study was to expand upon the preliminary study findings by addressing the identified limitations. Study aims were to (1) determine participant retention and relapse rates in the fully integrated treatment and (2) reduce anxiety, depression, and craving symptoms 
among participants. We hypothesized that MBRP which targets decreasing impulsivity, craving, stress and reactivity would positively affect retention and relapse rates and decrease reported depression, anxiety, and craving symptoms among MBRP/MOUD participants as compared to TAU/MOUD.

\section{Methods}

\section{Participants}

Participants were recruited from treatment as usual (TAU) MOUD clinic groups from October 2017 through December 2019. Eligible and interested participants met with the project investigators, who described the study, administered consent, and conducted baseline data assessments. Participants were given the option to enroll in MBRP plus MOUD or remain in TAU plus MOUD as would be expected in a typical treatment setting.

A total of 80 eligible participants were recruited into the study (MBRP/MOUD, $n=35$; TAU/MOUD, $n=45$ ). An intervention flow chart is provided in Fig. 1. Participant baseline demographics are located in Table 1 by group. For categorical characteristics, the two groups were compared using Fisher's exact test. No significant differences were detected at baseline between MBRP/MOUD and TAU/ MOUD participants within marital status $(p=0.63)$, gen$\operatorname{der}(p=0.12)$, employment $(p=0.95)$, education $(p=0.10)$, or insurance $(p=0.59)$. The overall sample mean age was
$36.3(S D=8.7)$. A $t$-test comparing groups in age revealed MBRP/MOUD group participants were statistically significantly $(p=0.02)$ younger $(M=34.9, S D=6.9)$ than TAU/ MOUD group participants $(M=37.3, S D=10.3)$. However, the effect size for this difference was small (Cohen's $d=0.21$ ), suggesting this difference was not practically important.

\section{Procedure}

Eligible participants were recruited during the intermediate stage of treatment (patients with at least 90 consecutive days substance free) from West Virginia University's Comprehensive Opioid Addiction Treatment (COAT) program. The intermediate phase of treatment is approximately 9 months in duration if the patient maintains stability, and includes every other week medication management in a group setting followed by cognitive behavioral-based group therapy. Patients are required to attend a minimum of four community-based self-help meetings (e.g., Narcotics Anonymous or SMART Recovery) weekly as part of their participation in treatment.

Typically, patients are prescribed between 2 and $16 \mathrm{mg}$ of buprenorphine/naloxone per day and participants continued to receive the medication at the dose determined by their COAT physician in both TAU/MOUD and MBRP/MOUD groups. Buprenorphine/naloxone is approved by the US Food and Drug Administration (FDA) for the treatment of opioid use disorder and was taken sublingually. Buprenorphine is a partial agonist medication and naloxone is an

Fig. 1 Intervention flowchart

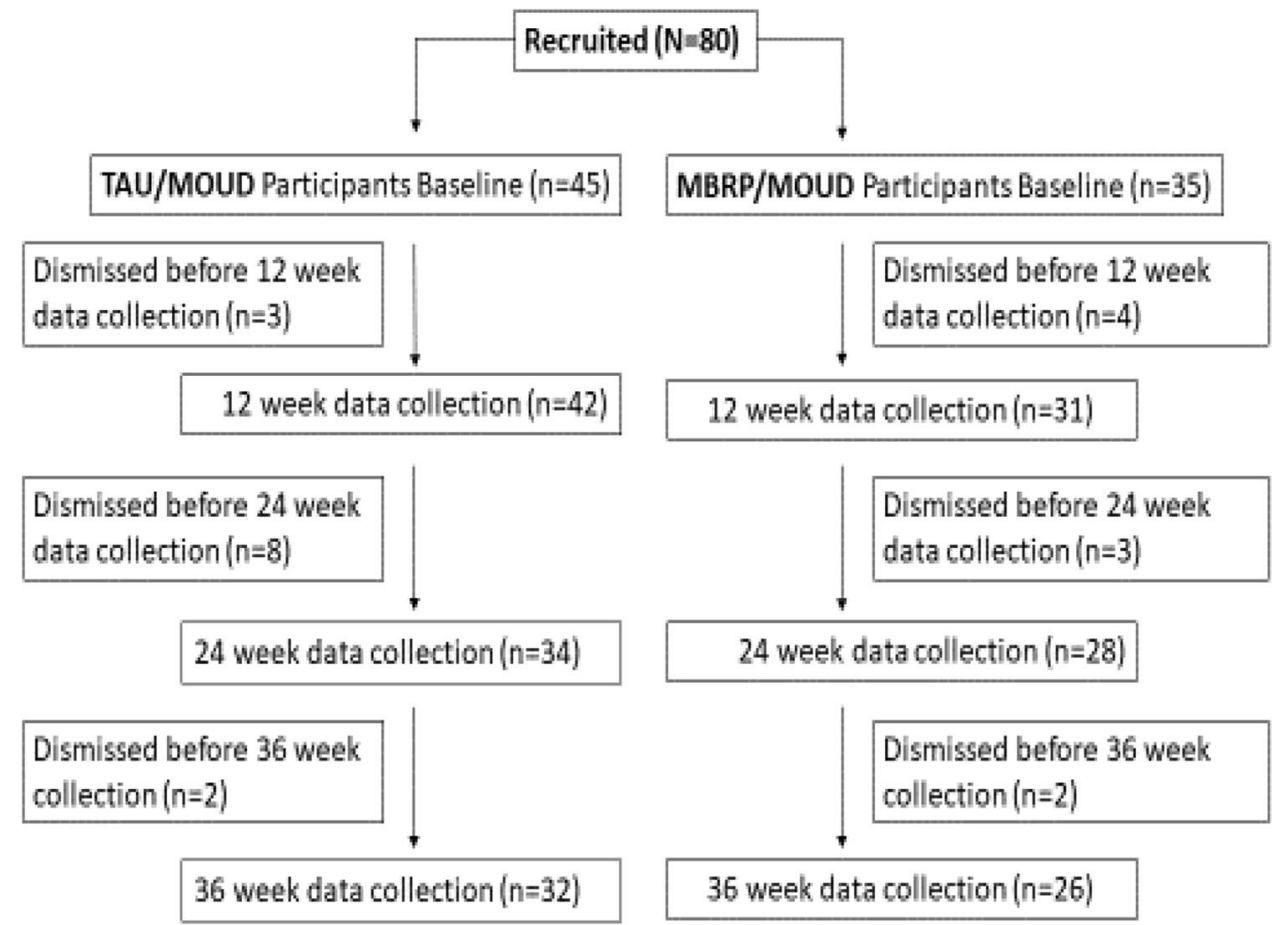


Table 1 MBRP intervention demographic data by group and total $(N=80)$

\begin{tabular}{|c|c|c|c|c|}
\hline Demographic & $\operatorname{MBRP}(n=35)$ & TAU $(n=45)$ & Total & $p$-value \\
\hline \multicolumn{5}{|l|}{ Marital status } \\
\hline & & & & .63 \\
\hline Single & 18 & 28 & 46 & \\
\hline Married & 8 & 8 & 16 & \\
\hline Divorced or separating & 9 & 9 & 18 & \\
\hline \multicolumn{5}{|l|}{ Gender } \\
\hline & & & & .12 \\
\hline Male & 13 & 24 & 37 & \\
\hline Female & 20 & 21 & 41 & \\
\hline Other & 2 & 0 & 2 & \\
\hline \multicolumn{5}{|l|}{ Race } \\
\hline & & & & $\mathrm{n} / \mathrm{a}$ \\
\hline White & 35 & 45 & 80 & \\
\hline \multicolumn{5}{|l|}{ Employment } \\
\hline & & & & .95 \\
\hline Full time & 13 & 18 & 31 & \\
\hline Part time & 7 & 8 & 15 & \\
\hline Unemployed & 15 & 19 & 34 & \\
\hline \multicolumn{5}{|l|}{ Education } \\
\hline & & & & .10 \\
\hline Did not finish high school & 5 & 3 & 8 & \\
\hline High school graduate/GED & 15 & 30 & 45 & \\
\hline Some college or greater & 16 & 11 & 27 & \\
\hline \multicolumn{5}{|l|}{ Insurance } \\
\hline & & & & .59 \\
\hline Medicaid & 31 & 34 & 65 & \\
\hline Medicare & 2 & 3 & 5 & \\
\hline Private & 3 & 7 & 10 & \\
\hline
\end{tabular}

opioid antagonist. The naloxone is only activated if the medication is used intravenously or intranasally. Study inclusionary criteria were having a diagnosis of opioid use disorder within the last 12 months as determined by DSM-5 criteria; being active in the intermediate stage of treatment in the COAT clinic; 90 consecutive days substance free at the time of recruitment and prior to starting the intervention; male or a non-pregnant, non-nursing female age 18 or greater; being able to understand and provide written informed consent to participate; being able to provide contact information; and being able to comprehend and communicate in English. Pregnant and nursing women were excluded from the study because they are treated in a separate pregnancy and postpartum clinic integrated with obstetrician and gynecologist care. Exclusionary criteria were assessed by the treating physician and study team and included being at high risk for suicide; at high risk for study drop out due to incarceration; having a current psychotic disorder or co-morbid diagnosis with psychotic features which would prevent the participant from engaging in the mindfulness intervention; and a primary substance use disorder other than opioid use disorder. Participants who may have become suicidal during the course of the study were evaluated by the treating physician and provided immediate intervention to ensure safety and stabilization. This study was approved by West Virginia University's Institutional Review Board (Protocol \# 1708720058R001).

\section{MBRP Intervention Modifications}

Two modifications to traditional MBRP were tested in the current study. First, participants in the MBRP/MOUD group were assigned to attend a 24-week, bi-weekly 60-min group therapy session led by a licensed therapist with specific certification in mindfulness interventions and trained in the manualized mindfulness-based relapse prevention. The role of the MBRP therapist was to teach mindfulness techniques through didactic and practical exercises so participants would see the transient nature of thoughts and emotions including symptoms of craving, depression, and anxiety. Sessions were informed by prior studies of mindfulness 
in the setting of substance abuse and relapse prevention, including mindfulness-based relapse prevention (Bowen et. al., 2006 and 2014).

Second, session format was expanded from an 8-week close-ended group in the pilot study (see Zullig et al., 2018 ) to a 12-week open-ended group (meeting once every 2 weeks for 24 weeks) for 12 total hours of programming. In the MBRP model, there are eight primary areas of focus, but instead of having each individual session focus on one topic, we integrated multiple topics into each session so that individuals could come and go into the group without missing an area of focus. The eight areas of focus were automatic pilot and relapse, awareness of triggers and craving, mindfulness in daily life, mindfulness in high-risk situations, acceptance and skillful action, seeing thoughts as thoughts, self-care and lifestyle balance, and social support and practice. Each skill was taught during the course of the 24 weeks, but it did not matter when participants entered into the group cycle. We also incorporated mindfulness "warm up" and orientation each time there was a new group member where more experienced group members shared their own knowledge and experience thus reinforcing concepts and solidifying learning. This adaptation was especially important for individuals with trauma histories as they may experience uncomfortable body sensations. Physical discomfort can trigger memories of previous trauma and result in participants wanting to discontinue the intervention if the pace moves too quickly. Once participants completed the 24-week MBRP/MOUD intervention, they were reintegrated into the TAU/MOUD therapy groups in the COAT clinic and were followed for 12 additional weeks.

\section{Measures}

Study outcomes included participant (1) retention in substance abuse treatment; (2) relapse on any prohibited substance; (3) time of relapse; (4) self-reported craving, anxiety, and depression levels; and (5) self-reported mindfulness. In the MBRP/MOUD group, participants were assigned to attend a bi-weekly 60 -min session led by a licensed therapist for 24-weeks. Measures were administered at baseline, 12 weeks, 24 weeks, and 36-weeks post-recruitment. All study participants received a $\$ 10$ gift card each time they completed the measures for a total of $\$ 40$ if they completed the study.

Retention Rate Retention was defined as remaining in MOUD in the TAU and 24-week MBRP intervention groups and was calculated again at 36 weeks. Participants who missed attendance at two consecutive COAT program sessions were not retained in bi-weekly treatment as per clinic policy regardless of group and therefore ineligible to be retained in the study. Participants who missed more than two groups in the 24 weeks were also not retained in the study so as to avoid dose effect issues. Treatment retention was calculated by dividing the number of participants retained at 24 and 36 weeks of TAU and MBRP by the total number of participants in each group and multiplying by 100 (range 0-100\%).

Rate of Relapse Relapse data was obtained from the electronic medical record. When participants presented for treatment, they were asked by the clinical team to report the number of days of sobriety and if they had relapsed, what substance they had relapsed on. Any non-prescribed substance use during treatment was considered a relapse, including alcohol. In addition to self-report, relapses were captured through urine drug screens conducted as part of standard MOUD treatment. If a participant reported use of more than one substance since their last clinic visit, this was counted as one relapse for each substance used. This information was recorded in the progress note. Data from progress notes was then extracted from the medical record and entered into the study database on a quarterly basis.

Craving Symptoms Craving symptoms were measured by the 14-item Desire for Drugs Questionnaire (DDQ), which has previously demonstrated acceptable validity and internal consistency reliability with estimates exceeding 0.80 (Franken et al., 2002). Response options are (a) strongly disagree, (b) disagree, (c) undecided, (d) agree, and (e) strongly agree with values from 1 (strongly disagree) to 5 (strongly agree) assigned. The sum of the response values in total (range 14-70) was the outcome of interest for cravings, with higher values indicative of greater cravings. Craving was measured at baseline, after 12 weeks, post-intervention (24 weeks), and again 36 weeks post-intervention among MBRP/MOUD and TAU/MOUD study participants. The baseline internal consistency estimate for the DDQ in this study was 0.78 .

Depression Depression was assessed using the 5-item Overall Depression Severity and Impairment Scale (ODSIS), a self-report continuous measure designed for use across heterogeneous mood disorders and with subthreshold depressive symptoms. Each ODSIS item has 4 response options that are summed. A total score of 8 or higher (range 0-20) is generally used to determine a depression diagnosis (correctly classifies over $80 \%$ ). The scale has demonstrated convergent and discriminant validity and internal consistency reliability with estimates exceeding 0.91 in clinical and non-clinical populations (Bentley et al., 2014). Depression was measured at baseline, after 12 weeks, post-intervention (24 weeks), and again 36 weeks post-intervention among MBRP/MOUD and TAU/MOUD study participants. The 
baseline internal consistency estimate for the ODSIS in this study was 0.89 .

Anxiety Anxiety was assessed using the 5-item Overall Anxiety Severity and Impairment Scale (OASIS), a selfreport measure that can be used to assess severity and impairment associated with any anxiety disorder or multiple anxiety disorders. Each OASIS item has 4 response options that are summed. A total score of 8 or higher (range $0-20$ ) is generally used to determine an anxiety diagnosis (correctly classifies over $80 \%$ ). The scale has demonstrated convergent and divergent validity and internal consistency reliability with estimates exceeding 0.80 in clinical (Campbell-Sills et al., 2009) and non-clinical (Norman et al., 2006) populations. Anxiety was measured at baseline, after 12 weeks, post-intervention ( 24 weeks), and again 36 weeks postintervention among MBRP/MOUD and TAU/MOUD study participants. The baseline internal consistency estimate for the OASIS in this study was 0.92 .

Mindfulness Mindfulness was measured with the 39-item self-report 5-Facet Mindfulness Questionnaire (FFMQ), which has demonstrated validity and adequate internal consistency with estimates ranging from 0.75 to 0.91 for the five subscales in prior research (Baer et al., 2006). The measure requests participants to best describe their opinion of what is generally true for each statement. Response options are (a) never or very rarely true, (b) rarely true, (c) sometimes true, (d) often true, and (e) very often or always true with values from 1 (never or very rarely true) to 5 (very often or always true). Because our interest was in determining whether participants in the MBRPMOUD group who became more mindful over time also displayed improved retention and relapse rates, and reported decreased depression, anxiety, and craving over time, the sum of the responses for the total scale divided by 5 (range 1-5) was the primary outcome of interest, with higher values indicating greater mindfulness. Mindfulness was measured at baseline, after 12 weeks, post-intervention ( 24 weeks), and again 36 weeks postintervention among MBRP/MOUD and TAU/MOUD study participants. The baseline internal consistency estimate for the FFMQ in this study was 0.89 with subscale estimates ranging from 0.79 for Non-Reactive to 0.92 for Describe.

\section{Data Analyses}

Descriptive statistics were used to summarize relapse and retention rates. A linear mixed model was performed on the self-report survey data and fixed effects are reported in the results. We selected the best model using the lowest Akaike information criterion (AIC). Linear mixed models (also called multilevel models) are a method for analyzing data that are non-independent, multilevel/hierarchical, and longitudinal, which allows us to explore the difference between effects within and between groups. In our case, we have participants receiving different treatments from different physicians and counselors. The data are random variables, and the parameters are random variables (at one level), but fixed at the highest level (for example, we still assume some overall population mean). AIC is an estimator of the relative quality of a statistical model where the lowest value is considered the best fitting model. Because MBRP/MOUD group participants were statistically significantly younger than TAU/MOUD participants, age was included in preliminary models and determined not to be a significant predictor for any of the scale or subscale analyses.

\section{Results}

\section{Relapse and Retention}

The overall retention rate of participants in treatment was $91 \%$ at 12 weeks, $78 \%$ at 24 weeks, and $73 \%$ at 36 weeks. This is higher than most studies of retention in MOUD report (Timko et al., 2016), likely because all of the participants were recruited from a clinical population stable in treatment with over 90 consecutive days of sobriety. Timko's systematic review (2016) identified retention rates in MOUD treatment with buprenorphine ranging from $46 \%$ to $66 \%$ at 6 months. Other studies have indicated a $65 \%$ retention rate for patients in this intermediate stage of treatment, 90-365 days (Zheng et al., 2017). Retention rates between the MBRP/MOUD group and TAU/MOUD group were similar. For example, the retention rate for the MBRP/MOUD group at 24 weeks was $80 \%$ and $76 \%$ for TAU/MOUD. At 36 weeks, the retention rate for the MBRP/MOUD group was $74 \%$ and $71 \%$ for TAU/MOUD.

With regard to relapse, $45 \%$ of all participants combined had at least one relapse during the 36 weeks; however, the rate of relapses for the MBRP/MOUD group was lower. At 24 weeks, the relapse rate for the MBRP/MOUD group was $31 \%$ and $40 \%$ for TAU/MOUD for participants reporting at least one relapse. At 36 weeks, the relapse rate for the MBRP/MOUD group was $43 \%$ and $47 \%$ for TAU/MOUD reporting at least one relapse. However, differences in the proportions were not significantly different between groups at 24 weeks $\left(\chi^{2}=0.63, p=0.43\right)$ or 36 weeks $\left(\chi^{2}=0.12\right.$, $p=0.73)$. Of all relapses reported, there were only four relapses on opioids (4\%), and two on tramadol. None of these medications was prescribed to the participant at the time of use. The majority of relapses were on alcohol, benzodiazepines, and methamphetamines (see Fig. 2). Qualitatively, a greater number of TAU/MOUD participants had more than one relapse $(n=14)$ compared to the MBRP/ MOUD group $(n=8)$. Of the participants who remained in 
Fig. 2 Number of relapses by substance at 36 weeks by group

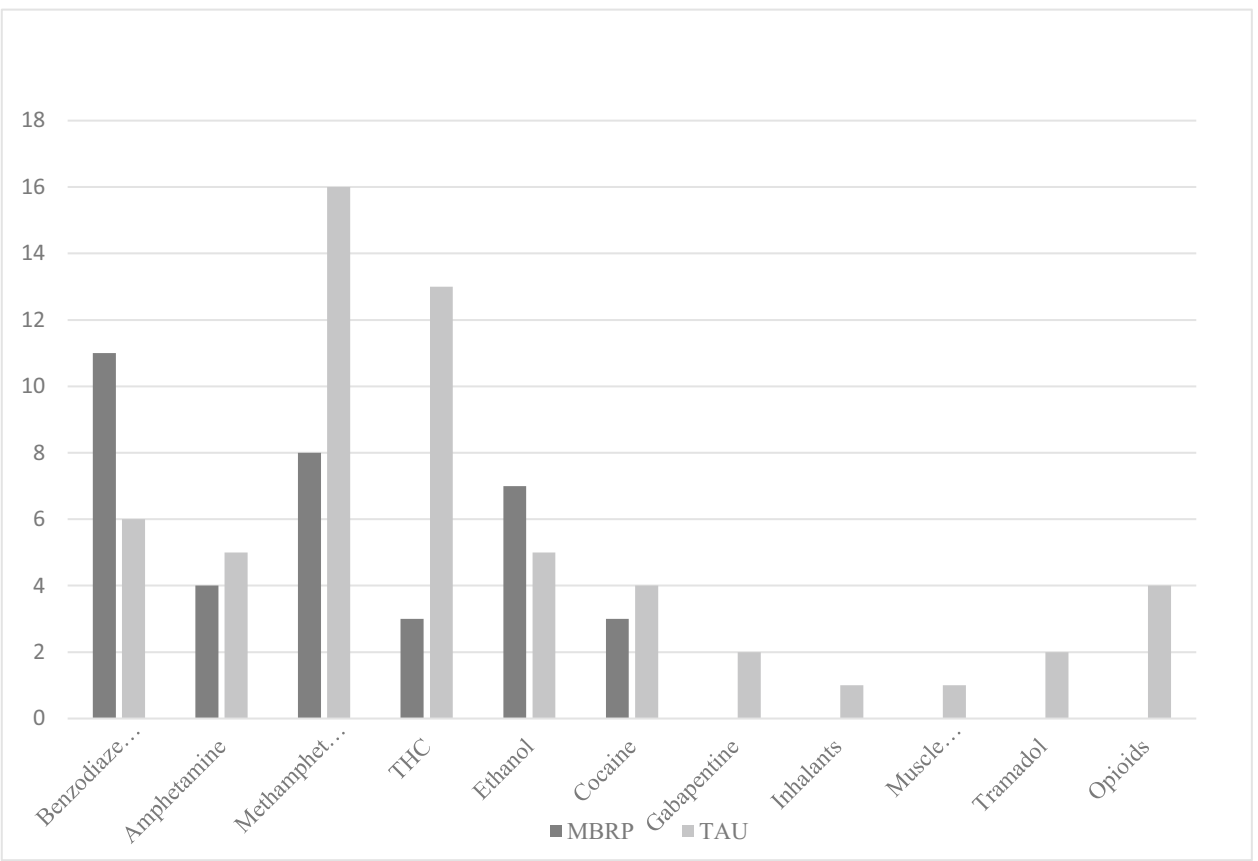

treatment the full 36 weeks, $19(60 \%)$ of the participants in TAU/MOUD and 17 (65\%) in MBRP/MOUD had no relapses.

\section{Craving}

Regression findings for self-reported craving are located in Fig. 3a. Analyses suggest that the change in reported mean craving over the course of the intervention was statistically significant $(F=2.66, p=0.02$ ). In other words, both groups reported significantly reduced craving over the course of the intervention, but there were no significant differences between the changes in the groups.

\section{Depression}

Regression findings for self-reported depression are located in Fig. 3b. Analyses suggest there were significant differences between groups in reported mean depression $(F=5.94$, $p<0.0001)$, with MBRP/MOUD participants reporting greater improvements over the course of the intervention. The MBRP/MOUD group reported greater mean depression at baseline compared to TAU; however, the MBRP/MOUD group also reported lower mean depression at the 12-, 24-, and 36-month assessments.

\section{Anxiety}

Regression findings for self-reported anxiety are located in Fig. 3c. Analyses suggest there were significant differences between groups in reported mean anxiety $(F=5.46$, $p<0.0001)$. The MBRP/MOUD group also reported greater mean anxiety at baseline compared to TAU; however, both groups reported similar downward trajectories in reported mean anxiety at 12-, 24-, and 36-month assessments, suggesting most of the differences between groups occurred between the baseline and 12-week assessments.

\section{Mindfulness}

Regression findings for self-reported mindfulness are located in Fig. 3d. Analyses suggest there were significant differences between groups in reported mean mindfulness $(F=14.50, p=0.0003)$ with MBRP/MOUD participants reporting greater improvements over the course of the intervention. The MBRP/MOUD group reported lower mean mindfulness at baseline compared to TAU/MOUD; however, the MBRP/MOUD group also reported greater mean mindfulness at the 12-, 24-, and 36-month assessments.

When examining the FFMQ subscales separately, significant differences between groups were detected for Observe $(p=0.006)$, Describe $(p=0.01)$, and Awareness $(p=0.002)$. However, there were no significant differences detected for Non-Reactive $(p=0.23)$, or Non-Judgement $(p=0.19)$.

\section{Supplementary Analysis}

Because there were significantly greater reductions in depression and anxiety in the MBRP/MOUD group and since the MBRP/MOUD group also reported greater levels of depression and anxiety at baseline, a supplementary analysis was performed to determine whether there was a 


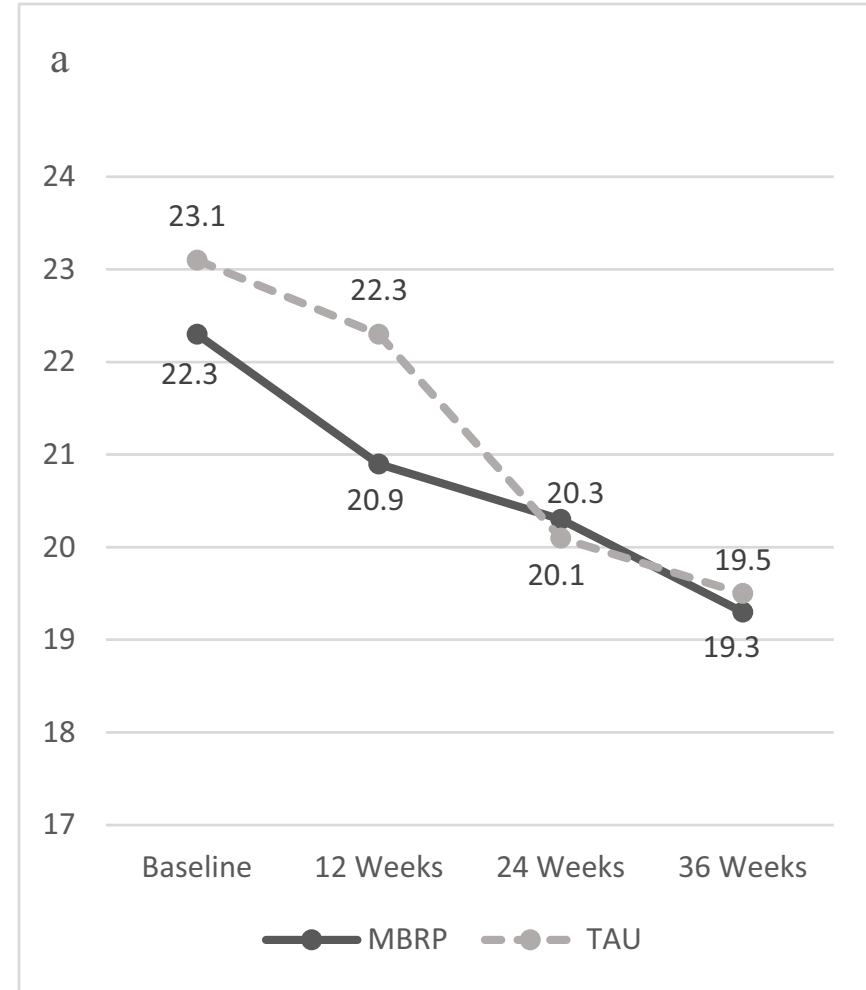

b
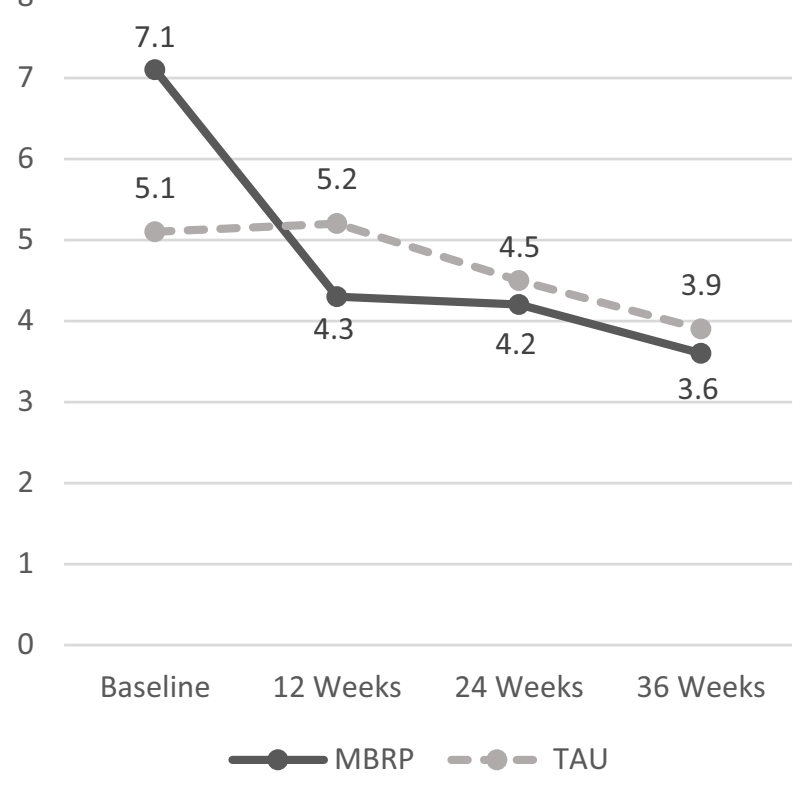

3.6

3.5

3.4

6.6

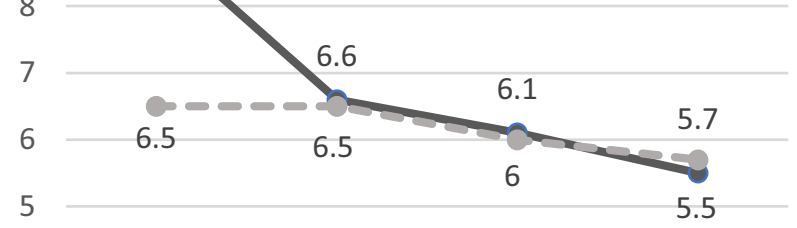

4

3

2

1

0

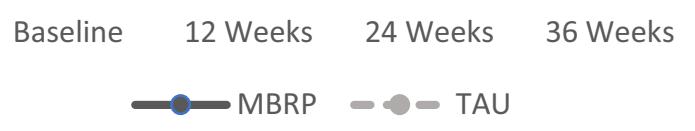

Fig. 3 Self-report mean scores by group across the 36-week intervention from the linear mixed model. a. Self-Report Mean Craving Scores Across Time (Range 14-70), b. Self-Report Mean Depression Scores Across Time (Range 0-20), c. Self-Report Mean Anxiety
8

3

2

1

0

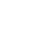

(1)

\section{d}

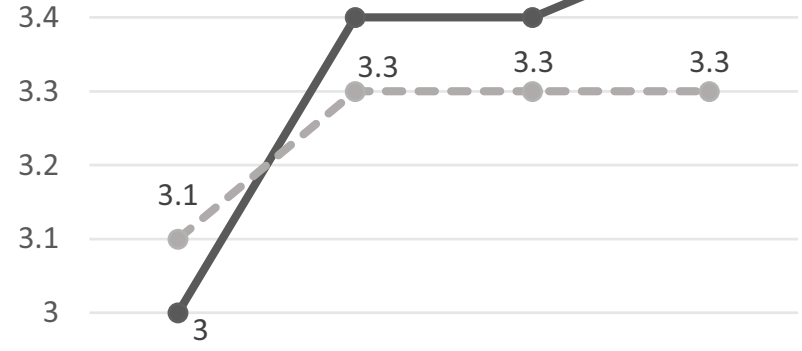

2.9

2.8

2.7

$$
\text { Baseline } 12 \text { Weeks } 24 \text { Weeks } 36 \text { Weeks }
$$

Scores Across Time (Range 0-20) and d. Self-Report Mean Mindfulness Scores Across Time (Range 1-5). Note: MBRP = MindfulnessBased Relapse Prevention group; TAU = Treatment As Usual group 
true intervention effect. This analysis showed correlations of 0.26 and 0.28 between craving at time 1 and the change in anxiety and depression scores, respectively, at time 2 for the overall sample. When analyzed separately by group, these correlations were smaller for the MBRP/MOUD group (0.14 and 0.07, respectively) compared to TAU/MOUD (0.29 and 0.36 , respectively), suggesting that craving was modestly associated with changes in mood in the TAU/MOUD group, but weakly in the MBRP/MOUD group.

\section{Discussion}

This study aimed to explore the effectiveness of MBRP with individuals receiving MOUD in a naturalistic, open-ended outpatient group treatment setting. Based on the preliminary work reported by Zullig et al. (2018), we made several modifications to the intervention, which included utilizing a quasi-experimental study design, increasing the sample size, and extending the follow-up period length to 12-weeks post intervention. In addition, we also increased the number of MBRP sessions from 8 to 12 held every other week over the 24-week intervention period to explore whether MBRP could be delivered in open-ended manner consistent with traditional outpatient treatment. Our overall hypothesis was that MBRP/MOUD would positively affect retention and relapse rates, increase reported mindfulness, and decrease reported depression, anxiety, and craving symptoms among participants.

Preliminary study results suggest that MBRP can be successfully implemented as an outpatient therapy with MOUD and that outcomes could be as good, if not better than treatment as usual. This is positive as integrating and adapting effective evidenced-based behavioral treatments into MOUD gives treatment providers additional treatment options for patients to optimize outcomes. For example, our study results suggest that significant decreases in craving were achieved in both MBRP/MOUD and TAU/MOUD groups. However, there were also significantly greater reductions in depression and anxiety in the MBRP/MOUD group indicating an intervention effect as reinforced by our supplementary analysis, despite the MBRP group reporting greater levels of depression and anxiety at baseline. Considering the fact that study participants had all been in weekly treatment for over 90 days prior to entering the study, these higher levels of depression and anxiety in the treatment group speak to the chronic nature of psychiatric disorders, including addiction, and the need for long-term interventions for the treatment of co-occurring mental health and substance use disorders. More importantly, these results suggest that those experiencing high levels of depression and anxiety may respond better to MBRP/MOUD than treatment as usual, especially since these reductions were achieved with concurrent and significantly greater increases in mindfulness in the MBRP/ MOUD group compared to TAU/MOUD 12 weeks postintervention. Lastly, although there were no statistically significant differences in relapse or retention rates between MBRP/MOUD and TAU/MOUD groups, higher numbers of participants experienced multiple relapses in TAU/MOUD compared to the MBRP/MOUD group.

Previous findings regarding the incidence of co-occurring mood disorders in the COAT clinic identified $72 \%$ of individuals who reported serious anxiety and $63 \%$ who reported serious depression (Winstanley et al., 2020). One of the salient findings of this study was that $43 \%$ of individuals in the MBRP/MOUD group and 49\% of individuals in the TAU/ MOUD group reported anxiety above the clinical threshold and $65 \%$ of individuals in the MBRP/MOUD group and $38 \%$ of the individuals in the TAU/MOUD group reported depression above the clinical threshold on their self-reported symptoms of both depression and anxiety. Of note is that these symptoms were still at clinically significant levels even after 90 days of treatment as usual. Untreated mood symptoms put patients at higher risk of relapse and treatment drop out increasing their chances of return to active use and overdose (Tolliver \& Anton, 2015). Hence, developing evidence-based interventions to address both substance abuse and mental health disorders concurrently is both an overdose prevention and treatment intervention tool. To this end, given the dramatic reductions in depression and anxiety levels from baseline to 12 weeks in the MBRP/MOUD group, results from the current study provide more robust evidence that MBRP may be a more effective intervention for populations with co-occurring mood and opioid use disorders. Given the significant differences between groups on the FFMQ subscales Observe, Describe, and Awareness, the acquisition of these skills may be a mechanism of change for improving mental health symptoms.

\section{Limitations and Future Research}

The current study is subject to several limitations. Although a quasi-experimental study design is an improvement over the pilot study's non-experimental study design, we cannot rule out selection bias given that participants were free to choose to enroll in the MBRP treatment or remain in TAU. In addition, the naturalistic design of implementing the study in the structure of an existing MOUD clinic setting was limiting because the study protocol then needed to follow the policies of the clinic so that the MBRP/MOUD and TAU/MOUD groups were comparable. This resulted in the inability to retain people in the study who were not retained in the bi-weekly phase of treatment. When patients were referred to a higher level of care due to relapse or missing two consecutive treatment sessions, they no longer met eligibility criteria for the study. The way that relapse data 
was collected in the study could be improved upon by using the Timeline Follow Back method (Sobell \& Sobell, 1996), which would have provided more information about relapse severity. Relapse for the study was defined to be consistent with the clinical setting we recruited from which limited our ability to assess for any differences in relapse severity in the two groups. The only statistically significant difference between groups at baseline was age, where MBRP/MOUD participants were younger than TAU/MOUD participants; however, age was not a significant predictor in any of the scale or subscale analyses. There were no other significant demographic differences between groups at baseline; however, those who enrolled in the MBRP/MOUD treatment reported substantially higher levels of depression and anxiety at baseline, perhaps because they believed TAU/MOUD after 90 days was not addressing their mental health symptoms adequately. Despite this dynamic, supplementary analysis determined craving weakly explained changes in mood in the MBRP/MOUD group. Quasi-experimental study designs are limited in their ability to conclusively establish causality; therefore although our results appear promising, we cannot definitively conclude that the positive reductions in depression and anxiety were caused by the MBRP treatment. Future studies utilizing random assignment to treatment and control conditions are necessary to address both of these limitations. Our study sample also identified primarily as White. Therefore, our results may not be generalizable to more racially diverse populations receiving MOUD for opioid use disorder in an outpatient setting. Lastly, our sample size left the study somewhat underpowered to be able to determine significant differences in the relapse and retention rates between the two groups.

Future directions include conducting a large-scale randomized clinical trial to identify any statistical difference in relapse and retention rates for participants in MBRP/ MOUD compared to TAU/MOUD since only trends supporting superior outcomes for MBRP were found in this small study. In addition, with the movement away from a one size fits all model towards more personalized medicine, a larger scale trial would serve to identify characteristics of patients who might benefit most from MBRP/MOUD. Overall, the findings add to the overwhelming support of the efficacy of MOUD in retention of patients and low relapse rates on opioids.

Author Contribution KJZ: led all aspects of the study, including writing of the article and data analyses. LRL: helped conceive the study, led study recruitment efforts, and provided input into the article. MT and MG: coordinated study recruitment and data collection efforts and provided input into the article. GRH: provided statistical support and input into the article. LF: co-led MBRP sessions with participants and provided input into the article.
Funding This research was supported by the US Centers for Disease Control and Prevention (Grant Number 6R49CE002109-05-06). Its contents are solely the responsibility of the authors and do not necessarily represent the official views of the Centers for Disease Control and Prevention or the Department of Health and Human Services.

\section{Declarations}

Ethics Approval and Consent to Participate Individual informed consent was obtained from all study participants. All procedures were performed in accordance with the ethical standards of the West Virginia University Institutional Review Board for human subjects research.

Conflict of Interest The authors declare no competing interests.

\section{References}

Ahmad, F. B., Rossen, L. M. \& Sutton, P. (2021). Provisional drug overdose death counts. National Center for Health Statistics, US Center for Disease Control and Prevention. Retrieved June 25, 2021 from https://www.cdc.gov/nchs/nvss/vsrr/drug-overdosedata.htm.

Andrilla, C. H. A., Moore, T. E., Patterson, D. G., \& Larson, E. H. (2019). Geographic distribution of providers with a DEA waiver to prescribe buprenorphine for the treatment of opioid use disorder: A 5-year update. The Journal of Rural Health, 35(1), 108112. https://doi.org/10.1111/jrh.12307

Baer, R. A., Smith, G. T., Hopkins, J., Krietemeyer, J., \& Toney, L. (2006). Using self-report assessment methods to explore facets of mindfulness. Assessment, 13(1), 27-45. https://doi.org/10.1177/ 1073191105283504

Bentley, K. H., Gallagher, M. W., Carl, J. R., \& Barlow, D. H. (2014). Development and validation of the Overall Depression Severity and Impairment Scale. Psychological Assessment, 26(3), 815830. https://doi.org/10.1037/a0036216

Bowen, S., Somohano, V. C., Rutkie, R. E., Manuel, J. A., \& Rehder, K. L. (2017). Mindfulness-based relapse prevention for methadone maintenance: A feasibility trial. JOurnal of Alternative and Complementary Medicine, 23(7), 541-544. https://doi.org/10. 1089/acm.2016.0417

Bowen, S., Witkiewitz, K., Dillworth, T. M., Chawla, N., Simpson, T. L., Ostafin, B., Larimer, M. E., Blume, A. W., Parks, G. A., \& Marlatt, G. A. (2006). Mindfulness meditation and substance use in an incarcerated population. Psychology of Addictive Behaviors, 20(3), 343-347. https://doi.org/10.1037/0893-164X.20.3.343

Bowen, S., Witkiewitz, K., Clifasefi, S. L., Grow, G., Chawla, N., Hsu, S. H., Carroll, H. A., Harrop, E., Collins, S. E., Lustyk, M. K., \& Larimer, M. E. (2014). Relative efficacy of mindfulness-based relapse prevention, standard relapse prevention, and treatment as usual for substance use disorders: A randomized clinical trial. JAMA Psychiatry, 71(5), 547-556. https://doi.org/10.1001/jamap sychiatry.2013.4546

Carroll, K., \& Weiss, R. (2017). The role of behavioral treatments in buprenorphine maintenance treatment: A review. American Journal of Psychiatry, 174(8), 738-747. https://doi.org/10.1176/appi. ajp.2016.16070792

Campbell-Sills, L., Norman, S. B., Craske, M. G., Sullivan, G., Lang, A. J., Chavira, D. A., Bystritsky, A., Sherbourne, C., Roy-Byrne, P., \& Stein, M. B. (2009). Validation of a brief measure of anxietyrelated severity and impairment: The Overall Anxiety Severity and Impairment Scale (OASIS). Journal of Affective Disorders, 112(1-3), 92-101. https://doi.org/10.1016/j.jad.2008.03.014 
Centers for Disease Control and Prevention (CDC, 2017). Opioid overdose: Drug overdose death data. Retrieved March 4, 2021 from https://www.cdc.gov/drugoverdose/data/statedeaths.html

Centers for Disease Control and Prevention (CDC), National Center for Health Statistics. Multiple Cause of Death 1999-2014 on CDC WONDER Online Database. (2015). Data are from the Multiple Cause of Death Files, 1999-2014, as compiled from data provided by the 57 vital statistics jurisdictions through the Vital Statistics Cooperative Program. Retrieved March 4, 2021 from http://wonder.cdc.gov/mcd-icd10.html

Franken, I. H. A., Hendriks, V. M., \& van den Brink, W. (2002). Initial validation of two opiate craving questionnaires: The Obsessive Compulsive Drug Use Scale and the Desires for Drug Questionnaire. Addictive Behaviors, 27(5), 675-685. https://doi.org/10. 1016/s0306-4603(01)00201-5

Garland, E. L. (2013). A mindfulness oriented recovery enhancement for addiction, stress, and pain. NASW Press.

Garland, E. L., Froeliger, B., Zeidan, F., Partin, K., \& Howard, M. O. (2013). The downward spiral of chronic pain, prescription opioid misuse, and addiction: Cognitive, affective, and neuropsychopharmacologic pathways. Neuroscience \& Biobehavioral Reviews, 37(10), 2597-2607. https://doi.org/10.1016/j.neubiorev.2013.08. 006

Garland, E. L., Gaylord, S. A., Boettiger, C. A., \& Howard, M. O. (2010). Mindfulness training modifies cognitive, affective, and physiological mechanisms implicated in alcohol dependence: Results of a randomized controlled trial. Journal of Psychoactive Drugs, 42(2), 177-192. https://doi.org/10.1080/02791072.2010. 10400690

Grant, S., Hempel, S., Colaiaco, B., Motala, A., \& SHanman, R.M., Booth, M., Dudley, W., Sorbero, M.E. . (2015). Mindfulnessbased relapse prevention for substance use disorder: A systematic review. Rand Corporation.

Gu, J., Strauss, C., Bond, R., \& Cavanagh, K. (2015). How do mindfulness-based cognitive therapy and mindfulness-based stress reduction improve mental health and wellbeing? A systematic review and meta-analysis of mediation studies. Clinical Psychological Review, 37, 1-12.

Hedegaard, H., Miniño, A.M., \& Warner, M. (2020). Drug overdose deaths in the United States, 1999-2018. NCHS Data Brief, no 356. Hyattsville, MD: National Center for Health Statistics. Retrieved March 4, 2021 from https://stacks.cdc.gov/view/cdc/ 84647

Ivtzan, I. (2020). Handbook of mindfulness-based programmes. Routledge.

Korecki, R.J., Schwebel, F.J., Votaw, V.R., \& Witkiewitz, K. (2020). Mindfulness-based programs for substance use disorders: A systematic review of manualized treatments. Substance Abuse Treatment, Prevention, and Policy. https://doi.org/10.1186/ s13011-020-00293-3

Larochelle, M., Bernson, D., Land, T., Stopka, T. J., Wang, N., Xuan, Z., Bagley, S. M., Liebschutz, J. M., \& Walley, A. Y. (2018). Medication for opioid use disorder after nonfatal opioid overdose and association with mortality: A cohort study. Annals of Internal Medicine, 169(3), 137-145. https://doi.org/10.7326/M17-3107

Norman, S. B., Cissell, S. H., Means-Christensen, A. J., \& Stein, M. B. (2006). Development and validation of an Overall Anxiety Severity and Impairment Scale (OASIS). Depression and Anxiety, 23(4), 245-249. https://doi.org/10.1002/da.20182

Penberthy, J. K., Konig, A., Gioia, C. J., Rodríguez, V. M., Starr, J. A., Meese, W., Worthington-Stoneman, D., Kersting, K., \& Natanya, E. (2015). Mindfulness-based relapse prevention: History, mechanisms of action, and effects. Mindfulness, 6(2), 151-158. https:// doi.org/10.1007/s12671-013-0239-1

Roos, C., Kirouac, M., Stein, E., Wilson, A., Bowen, S., \& Witkiewitz, K. (2019). An open trial of rolling admission mindfulness-based relapse prevention (Rolling MBRP): Feasibility, acceptability, dose-response relations, and mechanisms. Mindfulness, 10(6), 1062-1073. https://doi.org/10.1007/s12671-018-1054-5

Roos, C. R., Bowen, S., \& Witkiewitz, K. (2017). Baseline patterns of substance use disorder severity and depression and anxiety symptoms moderate the efficacy of mindfulness-based relapse prevention. Journal of Consulting and Clinical Psychology, 85, 1041-1051. https://doi.org/10.1037/ccp0000249

Sobell, L. C., \& Sobell, M. B. (1996). Timeline Follow Back user's guide: A calendar method for assessing alcohol and drug use. Toronto, Canada: Addiction Research Foundation.

Sordo, L., Barrio, G., Bravo, M. J., Indave, B. I., Degenhardt, L., Wiessing, L., Ferri, M., \& Pastor-Barriuso, R. (2017). Mortality risk during and after opioid substitution treatment: Systematic review and meta-analysis of cohort studies. British Medical Journal, 357, j1550. https://doi.org/10.1136/bmj.j1550

Substance Abuse and Mental Health Services Administration (SAMHSA, 2020). Key substance use and mental health indicators in the United States: Results from the 2019 National Survey on Drug Use and Health (HHS Publication No. PEP20-07-01001, NSDUH Series H-55). Rockville, MD: Center for Behavioral Health Statistics and Quality, Substance Abuse and Mental Health Services Administration.

Tolliver, B., \& Anton, R. (2015). Assessment and treatment of mood disorders in the context of substance abuse. Dialogues in clinical neuroscience, 17(2), 181-190. https://doi.org/10.31887/DCNS. 2015.17.2

Timko, C., Schultz, N. R., Cucciare, M. A., Vittorio, L., \& GarrisonDiehn, C. (2016). Retention in medication-assisted treatment for opiate dependence: A systematic review. Journal of Addictive Diseases, 35(1), 22-35. https://doi.org/10.1080/10550887.2016. 1100960

U.S. Congress. (2015). Recovery Enhancement for Addiction Treatment Act. Retrieved March 24, 2021 from http://www.markey.senate. gov/imo/media/doc/2015-05-27-TREAT-Act-BillText.pdf.

Winstanley, E. L., Lander, L. R., Berry, J. H., Mahoney, J. J., Zheng, W., Herschler, J., Marshalek, P., Sayres, S., Mason, J., \& Haut, M. W. (2020). West Virginia's model of buprenorphine expansion: Preliminary results. Journal of Substance Abuse Treatment, 108, 40-47. https://doi.org/10.1016/j.jsat.2019.05.005

Witkiewitz, K., Warner, K., Sully, B., Barricks, A., Stauffer, C., Thompson, B. L., \& Luoma, J. B. (2014). Randomized trial comparing mindfulness-based relapse prevention with relapse prevention for women offenders at a residential addiction treatment center. Substance Use \& Misuse, 49(5), 536-546. https://doi.org/ 10.3109/10826084.2013.856922

Witkiewitz, K., Bowen, S., Douglas, H., \& Hsu, S. H. (2013). Mindfulness-based relapse prevention for substance craving. Addictive Behaviors, 38(2), 1563-1571. https://doi.org/10.1016/j.addbeh. 2012.04.001

Zullig, K. J., Lander, L. R., Sloan, S., Brumage, M. R., Hobbs, G. R., $\&$ Faulkenberry, L. (2018). Mindfulness-based relapse prevention with individuals receiving medication-assisted outpatient treatment for opioid use disorder. Mindfulness, 9, 423-429. https:// doi.org/10.1007/s 12671-017-0784-0

Zheng, W., Nickasch, M., Lander, L., Wen, S., Xiao, M., Marshalek, P., Dix, E., \& Sullivan, C. (2017). Treatment outcome comparison between telepsychiatry and face-to-face buprenorphine medication-assisted treatment for opioid use disorder: A 2-year retrospective data analysis. Journal of Addiction Medicine, 11(2), 138-144. https://doi.org/10.1097/ADM.0000000000000287

Publisher's Note Springer Nature remains neutral with regard to jurisdictional claims in published maps and institutional affiliations. 